\section{Regarding “Aneurysm Rupture Following Treatment with Flow-Diverting Stents: Computational Hemodynamics Analysis of Treatment"}

$\mathrm{We}^{1}$ read with great interest the recent publication by Cebral et al entitled "Aneurysm Rupture Following Treatment with Flow-Diverting Stents: Computational Hemodynamics Analysis of Treatment." The postprocedural rupture of previously unruptured aneurysms after flow diversion (FD) is an uncommon but devastating complication. Correspondingly, any analysis that would allow operators to identify aneurysms at high risk for rupture after FD could improve the safety profile of the treatment strategy.

Cebral et $\mathrm{al}^{1}$ used a computational fluid dynamics (CFD) model to generate results, which suggest that FDs, in selected cases, may induce dramatic elevations of intra-aneurysmal pressure leading to postprocedural rupture. The authors further suggested that their numeric simulations would allow them to prospectively identify those aneurysms at risk for rupture after FD. They present 7 aneurysms, 3 with postprocedural rupture and 4 that were treated successfully. The selected aneurysms and arterial segments, along with assumptions about flow through the segments, constant pressure at the outlet of the segments, and rigid arterial walls, were evaluated within a CFD model. Using this model, their calculations showed that all 3 aneurysms which went on to rupture after FD demonstrated severe increases in intra-aneurysmal pressure $(>20 \mathrm{~mm} \mathrm{Hg})$ after treatment, while those aneurysms that did not rupture after FD did not exhibit such dramatic pressure rises $(<3 \mathrm{~mm} \mathrm{Hg}$ ).

While this hypothesis is very attractive, the modeling upon which these conclusions are based, seems flawed. The article underscores the general need to critically evaluate the results and carefully parse the pictures output by CFD programs, especially when pressures are reported. Given that the concepts are presented within the context of a complex mathematical argument, it may be difficult for readers who are inexperienced in fluid mechanics or CFD to fully appreciate the details of what the authors are proposing.

The major problems with the study include the following: 1) a vast overestimation of the physiologic pressure gradients occurring across short, tapered, and/or tortuous vascular segments; and 2) a selective invocation of cerebral autoregulation.

\section{The Baseline Pressure Gradient Values in the Cebral Model Are Incorrect}

A computational domain, which is usually a truncated segment of a vascular bed that is used to model hemodynamics, requires the specification of inlet and outlet flow conditions as well as the interaction of the fluid with the confining vascular geometry. Because the actual flow rates into the selected segments were not measured, they were calculated to generate an average (over the cardiac cycle) wall shear stress at the inlet of 15 dyne $/ \mathrm{cm}^{2}$. The authors also elected to set the outlet pressure to zero throughout the cardiac beat. The inlet pressure was then calculated by using the Navier-Stokes equations. Pressure distributions were then obtained by increasing the systolic input pressure to $120 \mathrm{~mm} \mathrm{Hg}$, while they also increased the output pressure to a nonzero value such that the pressure drops through the domain remained unchanged. The authors elected not to model the distensibility of the artery (ie, they neglected the important windkessel effect when attempting to predict pressure drops). Previous studies have shown and the authors themselves note that using rigid walls can result in unrealistic pressure gradients across the do-

Indicates open access to non-subscribers at www.ajnr.org main. ${ }^{2,3}$ Nonetheless, applying this set of assumptions to the modeled vascular segments, the CFD computations calculated pressure drops across these short vascular segments ranging between 20 and $40 \mathrm{~mm} \mathrm{Hg}$. These computations are vast overestimates that are in direct conflict with in and ex vivo experimental data as well as conventional fluid mechanics.

Banerjee and $\mathrm{Back}^{3}$ measured the pressure gradient that is required to drive blood through a $5.2-\mathrm{cm}$ segment of the canine femoral artery in vivo (3.8- $\mathrm{mm}$ diameter with a slight taper) under physiologic conditions of flow approximating those found in the internal carotid artery of humans (ie, the values of the Reynolds and Womersley numbers are equivalent and similar to those applied by Cebral et $\mathrm{al}^{1-3}$ ) In these experiments, Banerjee and Back measured a cyclic peak pressure drop of approximately $4.3 \mathrm{~mm} \mathrm{Hg}$. This pressure drop is attributable to the combination of the pressure required to overcome viscous dissipation (the cycle average pressure drop, approximately $0.6 \mathrm{~mm} \mathrm{Hg}$ ) and inertia during acceleration to peak systole (approximately $3.7 \mathrm{~mm} \mathrm{Hg}$ ). Thus, these experimentally measured pressure drops are almost an order of magnitude smaller than those calculated by Cebral et al.

The pressure drop across a vascular segment of known dimensions can be calculated by using basic principles of conventional fluid mechanics as well—namely, the Poiseuille calculations for the viscous pressure drop and Newton's second law for the pressure drop required to overcome inertia. Over a vascular segment with dimensions similar to those described by Banerjee and Back $^{3}$ and Cebral et al, ${ }^{1}$ these calculations yield 0.51 and $3.12 \mathrm{~mm} \mathrm{Hg}$, respectively, for viscous dissipation and inertial pressure drop, for a total pressure drop of 3.63 $\mathrm{mm} \mathrm{Hg}$. Thus, values calculated by using the basic principles of fluid mechanics under ideal flow conditions, while somewhat lower (by $16 \%$ ) than the measured experimental values mentioned, are nevertheless within a range of reasonable physiologic variance and again are almost an order of magnitude lower than those calculated by Cebral et al.

\section{Differing Mechanisms are Employed to Obtain Uniformity Among the Ruptured Aneurysms after FD}

According to the authors, the intra-aneurysmal pressure increases were caused by 1 of 2 different mechanisms:

\section{Mechanism 1}

Patients 1 and 3. The authors assume that the prestent arterial configuration proximal to the aneurysm created an enormous resistance to flow (and therefore a marked pressure drop) that was substantially relieved by the placement of the FD, such that the higher pressure at the inlet propagated further downstream and into the aneurysm.

In patient 1 , a mild (50\%) stenosis proximal to the aneurysm was resolved by placement of the FD. The dilation of this $50 \%$ stenosis after FD placement resulted in a calculated ("virtual") reduction in the trans-segmental pressure drop from 25 to $5 \mathrm{~mm} \mathrm{Hg}$. The authors suggested that this marked reduction in the trans-segmental pressure drop of $20 \mathrm{~mm} \mathrm{Hg}$ resulted in higher pressures over the entire parent artery segment and that these higher pressures were transmitted directly to the aneurysm fundus. They state, "As a consequence [of the preaneurysm stenosis dilation], the intra-aneurysmal pressure was increased by $20 \mathrm{~mm}$ Hg."

According to basic hemodynamic principles, the removal of a $50 \%$ stenosis reduces segmental resistance only minimally. Based on previous experiments using vessels with dimensions analogous to those described by the authors, removal of a $65 \%$ stenosis results in a reduc- 
tion in the trans-segmental peak pressure drop of half the value reported by the authors. ${ }^{4,5}$ Therefore, the calculations performed by Cebral et $\mathrm{al}^{1}$ result again in an overestimation of the trans-segmental pressure drop by at least a factor of 2 compared with traditional hemodynamic calculations and measurements. The authors opine that "This effect is well-known by endovascular specialists and is readily understandable." We speculate that they might have difficulty finding an experienced endovascular interventionist who would predict a 20 $\mathrm{mm} \mathrm{Hg}$ reduction in the trans-segmental pressure drop as a result of the dilation of a mild focal pre-aneurysmal stenosis.

In patient 3 , the authors attribute a large baseline pressure drop of $45 \mathrm{~mm} \mathrm{Hg}$ to parent artery tapering proximal to the aneurysm and a poststent pressure drop of approximately $20-25 \mathrm{~mm} \mathrm{Hg}$ to a sharp turn distal to the aneurysm. If such a high pressure drop could be attributed to 1 arterial segment, the heart would have to generate super-physiological pressures to overcome all of the sharp arterial turns and vascular taperings that occur between the aortic outlet and through the tortuous cerebrovasculature to reach the capillary outflow. As in patient 1 , the authors noted that this pre-existing taper was improved after FD placement. Thus, on the basis of these minimal changes in the vessel diameter after treatment, the authors calculate a profoundly exaggerated effect on the posttreatment pressures.

In evaluating the accuracy of the proposed calculations of Cebral et $\mathrm{al},{ }^{1}$ we can once again consider previous experimental studies and conventional fluid mechanics on arterial tapers ${ }^{6}$ and turns ${ }^{7}$ as a reference point for physiologically relevant pressure drops. For a $33 \%$ reduction in the diameter between an inlet and outlet of a $6-\mathrm{cm}$-long artery, the viscous pressure drop increases by $15 \%$ due to the taper compared with the Poiseuille value. ${ }^{6}$ There will be an additional pressure drop due to the change in momentum of approximately $4 \mathrm{~mm}$ $\mathrm{Hg}$ (derived from the Bernoulli equation). Then, the pressure drop required to overcome inertia in the vessel segment during acceleration to peak systole needs to be considered. Adding these 3 components, the total pressure drop across a "significantly" tapered artery would be around $5.6 \mathrm{~mm} \mathrm{Hg}$, which, once again, is an order of magnitude lower than the $45 \mathrm{~mm} \mathrm{Hg}$ value proposed by Cebral et al. In calculating the flow through pipes, the energy losses attributable to turns are classified as minor losses and (for a $180^{\circ}$ turn) are estimated to be approximately $20 \%$ of the kinetic energy of the fluid ${ }^{7}$; for blood under the proposed conditions, this value would be insignificant.

\section{Mechanism 2}

Patient 2. The authors assume that the implantation of the FD construct caused a significant increase in the resistance of the treated vascular segment, resulting in a subsequent reduction in flow. At this point, they selectively invoke "autoregulatory mechanisms" to raise the inlet pressure (ie, the systemic blood pressure) to maintain a constant flow rate through the segment. This "flow diverter-induced systemic hypertension" is then assumed to be transmitted directly to the aneurysm.

These are perhaps the most questionable assumptions in the entire study. The authors report that after the implantation of the FD construct, the pressure gradient required to drive equivalent flow though the analyzed segment increased from 20 to $48 \mathrm{~mm} \mathrm{Hg}$ ! They attributed this marked increase in the trans-segmental resistance to the tortuosity and tapering of the vascular segment being exacerbated by the implantation of the FD construct and to the "removal" of the aneurysm from the vascular circuit.

First, with respect to the effect of the FD on the segmental vascular resistance, such an increase in the trans-segmental pressure drop over a domain only a few centimeters in length, whether or not it includes an aneurysm, would be an impossibility from either a physiologic or hemodynamic standpoint. From a hemodynamic standpoint, the only way that the pressure would increase so dramatically would be for viscous dissipation and inertial forces to be tremendously increased after FD placement. Even if we assume that 2 FDs were placed concentrically inside each other inside the treated segment causing a luminal loss equal to approximately $200 \mu \mathrm{m}$ of cross-sectional diameter, the increases in pressure required to overcome the minimal stenotic effects of the FDs on the luminal cross-sectional area would be trivial. They can be calculated by using the Poiseuille and Newton laws to be $0.025 \mathrm{~mm} \mathrm{Hg}$ for viscous dissipation and $0.030 \mathrm{~mm} \mathrm{Hg}$ for inertial resistance or a total of $0.055 \mathrm{~mm} \mathrm{Hg}$ increase in peak pressure.

Second, the authors suggest that the placement of an FD results in the removal of the "low resistance" aneurysm from the vascular circuit, which now requires that all of the blood flow be shunted through the "higher resistance" reconstructed parent artery. They illustrate this "circuit" in Fig 5 that shows 2 parallel unlabeled individual resistors -1 representing the short arterial treated segment and the other representing the aneurysm itself. In analogy to basic electric circuits, hydraulic resistance is defined as pressure drop divided by the through-flow. Because flow has to enter and leave the aneurysm through the neck, it is reasonable to expect that the pressure gradient between the proximal and the distal side of the neck is zero or very close to zero. Therefore, the resistance to flow into and out of the aneurysm is nearly zero. However, even if the aneurysm "shunt" is removed and hypothetically replaced by a healthy blood vessel segment or a stent graft, only the "high resistance" over a short 2-cm segment would remain. The peak pressure drop over such a short segment can be estimated as approximately $40 \%$ of the pressure drop measured by Banerjee and Back ${ }^{3}$ over a longer segment of $5.2 \mathrm{~cm}^{2,3}$; using linear interpolation, the peak pressure drop would then be approximately $1.6 \mathrm{~mm} \mathrm{Hg}$. Because the aneurysm is not completely blocked by the FD, the total resistance through the parallel branches of the FD and the aneurysm would actually be less than that through the arterial segment alone; therefore, the actual peak pressure drop would likely be even lower than $1.6 \mathrm{~mm} \mathrm{Hg}$.

This profound increased resistance and exaggerated pressure drop after flow diversion is the opposite of the effects observed for patients 1 and 3, and runs counter to the proposed theory of elevated intraaneurysmal pressure leading to rupture. For this case to conform to the "elevated intra-aneurysmal pressure" theory, it was necessary to substantially increase the inlet pressure through a "complex system of autoregulation" to maintain cerebral blood flow. This assumption of "flow diverter-induced systemic hypertension" then makes the aneurysm of Patient 2 conform to the elevated intra-aneurysmal pressure theory. After the authors apply the assumption that the systemic blood pressure increases by $25 \mathrm{~mm} \mathrm{Hg}$ to compensate for this elevated trans-segmental resistance and reduced flow, they are able to conclude that the intra-aneurysmal pressure increases by a similar amount.

That the implantation of an FD induces an increase in the systemic blood pressure of $25 \mathrm{~mm} \mathrm{Hg}$ would seem to be easily measurable by the operators, their anesthesiologists, and critical care support staff. The blood pressure tracings from the peri-procedural period could be presented to validate such a claim.

Interestingly, their initial analysis demonstrated only a $2 \mathrm{~mm} \mathrm{Hg}$ increase in intra-aneurysmal pressure following FD. This negligible rise in intra-aneurysmal pressure following FD required the invocation of an "autoregulatory" mechanism to result in an elevated post- 
treatment intra-aneurysmal pressure. Contrarily, when the calculations in Patients 1 and 3 resulted in the opposite problem (a reduced pressure drop across the reconstructed segment due to the removal of a stenosis or tapering), systemic autoregulation as a mechanism for maintaining pre-FD perfusion was not used.

\section{Summary}

The conclusions presented by Cebral et $\mathrm{al}^{1}$ are based upon results that are not only largely inconsistent with both existing experimental data and basic fluid mechanics but are selectively applied to achieve a consistent conclusion for a group of aneurysms with a known outcome (postprocedural rupture). It is important to recognize that the results or conclusions presented in the manuscript have not been validated by either dynamic angiographic data or direct physiologic measurements. These results are solely the product of mathematic simulations that are only as valid as the assumptions on which they are based. As such, we would urge the readership to exercise extreme caution before incorporating any of the concepts proposed by Cebral et al, into clinical decision-making (eg, in the selection of patients to be treated with flow-diverting devices) or into the design of clinical research studies.

Disclosure: Drs Fiorella and Woo are unpaid consultants and proctors for Chestnut Medical/eV3. Dr Lieber is a stockholder in Surpass Medical Inc.

\section{References}

1. Cebral JR, Mut F, Raschi M, et al. Aneurysm rupture following treatment with flow-diverting stents: computational hemodynamics analysis of treatment. AJNR Am J Neuroradiol 2010;32:27-33. Epub 2010 Nov 11

2. Roy AS, Back LH, Banerjee RK. Evaluation of compliance of arterial vesse using coupled fluid structure interaction analysis. MCB Molecular and Cellular Biomechanics 2008;5:229-46

3. Banerjee RK, Back LH. Computed and measured hemodynamics in a compliant tapered femoral artery. In: Proceedings of the 2003 Summer Bioengineering Conference, Key Biscayne, Florida. June 25-29, 2003:261-62

4. Young DF. Fluid mechanics of arterial stenoses. J Biomech Eng 1979;101:157-75

5. Young DF, Cholvin NR, Roth AC. Pressure drop across artificially induced stenoses in the femoral arteries of dogs. Circ Res 1975;36:735-43

6. How TV, Black RA, Annis D. Comparison of pressure losses in steady nonNewtonian flow through experimental tapered and cylindrical arterial prostheses. J Biomed Eng 1988;10:225-30

7. Chandran KB, Yoganathan AP, Rittgers SE. Unsteady flow and nonuniform geometric models. In: Chandran KB, Yoganathan AI, Ritters ER, eds. Biofluid Mechanics: The Human Circulation. Boca Raton, Florida: Taylor \& Francis; 2006

D. Fiorella

C. Sadasivan

H. H. Woo

B. Lieber

Department of Neurosurgery

Stony Brook University Medical Center

Stony Brook, New York 